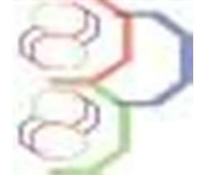

Journal of Applied Biosciences 151: 15540 - 15549

ISSN 1997-5902

\title{
Effet de la combinaison des fertilisants organiques et minéraux (NPK 15-15-15 et urée) sur le rendement de la laitue (Lactuca sativa $L$.) dans le sud du Togo
}

\author{
TCHANILEY Larounga ${ }^{1 *}$, AYISAH Kwasi Dzola², DEWA KASSA Kodjo Akonta ${ }^{3}$, \\ ${ }^{1}$ Laboratoire de Recherche sur les Agroressources et la Santé Environnementale (LARASE) Département de \\ Phytotechnie et d'amélioration des plantes. Ecole Supérieure d'Agronomie, Université de Lomé, 01 BP. 1515 Lomé, \\ Togo. \\ 2Laboratoire Interface Sciences du Sol, Climat et Production Végétale (LISSCPV). Département de Phytotechnie et \\ d'amélioration des plantes. Ecole Supérieure d'Agronomie, Université de Lomé, 01 BP. 1515 Lomé, Togo. \\ 3Institut de Conseil et d'Appui Technique (ICAT) Lomé, Togo \\ *Correspondant : tchanileadam@yahoo.fr tél (+228) 90257344
}

Original submitted in on $11^{\text {th }}$ May 2020. Published online at www.m.elewa.org/journals/ on $31^{\text {st }}$ July 2020

https://doi.org/10.35759/JABs.151.3

\section{RESUME}

Objectif : L'essai a visé à développer de nouvelles initiatives de gestion de la fertilité des sols compatibles avec la gestion rationnelle des ressources mises en jeux afin d'évaluer et d'apprécier l'efficacité des différentes combinaisons d'engrais sur le rendement de la laitue et d'identifier le meilleur model d'amélioration des conditions de culture de cette plante.

Méthodologie et résultats : Au cours de l'essai, le dispositif expérimental adopté a été celui en blocs aléatoire complet à quatre traitements et à trois répétitions. Les traitements utilisés ont été : T0 (témoin absolu sans apport de fertilisants), T1 (16 t.ha-1 de fumier de bovin +150 Kg.ha-1 de NPK:15-15-15 +50 Kg.ha-1 d'Urée), T2 (16 tha- ${ }^{-1}$ de fiente de volailles $+150 \mathrm{Kg}^{-h a^{-1}}$ de NPK :15-15-15 +50 Kg.ha-1 d'Urée) et T3 (16 tha-1 d'un mélange équitable de fientes de volailles et de fumier de bovin $+150 \mathrm{Kg}^{-h^{-1}}{ }^{-1}$ de NPK :1515-15 +50 Kg.ha-1 d'Urée). Les résultats obtenus ont montré que le traitement T2 a amélioré le rendement de la laitue qui a dépassé le témoin de $134 \%$.

Conclusion et application de résultats : L'étude a consisté à faire une évaluation de l'impact de différentes combinaisons d'engrais organiques et minéraux sur la productivité de la laitue. Les résultats obtenus ont monté que le traitement $\mathrm{T} 2$ suivi du traitement T3 puis du traitement $\mathrm{T} 1$ ayant donné respectivement un surplus de produit de $134,00 \%, 75,69 \%$ et de $62,16 \%$ par rapport au traitement témoin T0. La laitue étant une plante à cycle court, l'utilisation du fumier, à base de fientes de volailles, riche en azote dont la libération est rapide, serait mieux indiquée pour sa culture en plein champ et surtout en maraîchage.

Mots clés : laitue, fertilisation, combinaison, rendement 
Effect of the combination of organic and mineral fertilizers (NPK 15-15-15 and urea) on the yield of lettuce (Lactuca sativa L.) in southern Togo

\section{ABSTRACT}

Objective: The aim of the trial was to develop new soil fertility management initiatives compatible with the rational management of the resources involved in order to assess and assess the effectiveness of the different combinations of fertilizers on the yield of lettuce and to identify the best model for improving the growing conditions of this plant.

Methodology and results: During the test, the experimental device adopted was that in complete random blocks with four treatments and three repetitions. The treatments used were: T0 (absolute control without addition of fertilizers), T1 (16 tha- 1 of bovine manure $+150 \mathrm{Kg}$.ha- 1 of NPK: $15-15-15+50 \mathrm{Kg}$.ha-1 of Urea), T2 (16 tha-1 of poultry droppings $+150 \mathrm{Kg} \cdot \mathrm{ha}-1$ of NPK: $15-15-15+50 \mathrm{Kg}$.ha-1 of Urea) and T3 (16 tha -1 of a fair mix of poultry droppings and bovine manure $+150 \mathrm{Kg}$.ha-1 of NPK: $15-15-15+50 \mathrm{Kg}$.ha-1 of Urea). The results obtained showed that the T2 treatment improved the yield of the lettuce, which exceeded the control by $134 \%$.

Conclusion and application of results: The study consisted of assessing the impact of different combinations of organic and mineral fertilizers on the productivity of lettuce. The results obtained showed that the T2 treatment followed by the T3 treatment then by the T1 treatment having given respectively a surplus of product of $134.00 \%, 75.69 \%$ and $62.16 \%$ compared to the control treatment T0. Since lettuce is a short-cycle plant, the use of manure, based on poultry droppings, rich in nitrogen and quick to release, would be better suited for its cultivation in the open field and especially in market gardening.

Keywords: lettuce, fertilization, combination, yield

\section{INTRODUCTION}

Sous leurs diverses formes, les laitues sont les légumes salades les plus importants, disponibles toute l'année selon les variétés. Toutes les laitues sont issues de l'espèce sauvage Lactuca serriola qui pousse spontanément dans les clairières, les pentes rocailleuses et les terrains vagues. La laitue (Lactuca sativa) est une plante herbacée appartenant au genre Lactuca, de la famille des Astéracées, largement cultivée pour ses feuilles tendres consommées comme légume, généralement crues en salade. La laitue la plus cultivée est l'espèce Lactuca sativa à partir de laquelle les jardiniers ont sélectionné de nombreuses variétés et cultivars. La laitue est réputée pour ses vertus soporifiques, notamment sur les lapins, ce qui en fait un légume apprécié des jardiniers pour le potage. Cependant, la culture de la laitue (Lactuca sativa) se heurte à quelques problèmes d'adaptation dans certaines zones agro-écologiques. En zone tropicale chaude par exemple, la quantité et la qualité de la production se trouvent généralement et essentiellement affectées par une forte pression parasitaire liée aux conditions climatiques extrêmes. A ces problèmes s'ajoute l'utilisation excessive des engrais chimiques qui polluent les nappes phréatiques et entravent la fertilité des sols ainsi que la qualité des produits avec des effets néfastes sur la santé humaine, animale et végétale. C'est ainsi que pour palier à ces maux et diminuer davantage la quantité des engrais de synthèse utilisée sous la laitue, l'essai est menée à la Station d'Expérimentations Agronomiques de Lomé sur l'utilisation des fertilisants essentiellement organiques en vue d'améliorer la production de la laitue. De culture facile, la laitue est à adopter (Koudigué, 2018) En Afrique subsaharienne, la production agricole a énormément baissée pour la plupart des cultures maraîchères. La cause majeure de cette baisse est la diminution du niveau de fertilité des sols (Amadji et al. 2009). La faible productivité des cultures serait due à plusieurs contraintes dont la pauvreté des terres agricoles à cause d'une faible utilisation d'engrais minéraux et organiques, étant donné que l'insuffisance d'azote dans le sol a un impact direct sur la production (Macauley et Ramadjita, 2015). Au Togo, la tendance se tourne, 
sur les espaces maraichères, vers l'utilisation des engrais organiques à base des ordures ménagères et des émondes de Légumineuses arbustives fixatrices d'azote atmosphérique (Ayébou, 2002, Dansou 2003, Amouzouvi et al., 2005). Plusieurs travaux ont mis en évidence le gain quantitatif et qualitatif apporté par le fumier de ferme sur les sols cultivés en combinaison avec des fertilisants minéraux (IFDC, 2013). L'une des stratégies pour l'amélioration de la fertilité des sols, en particulier les sols ferralitiques reconnus pour leur pauvreté en azote et en phosphore est l'utilisation de compost (Koné et al., 2009. Biaou et al., 2017). Une bonne pratique agricole qui implique l'utilisation des composts produits avec les déchets organiques améliore les propriétés des sols, rend disponible des nutriments dans le sol et réduit les risques de pollution (Weber et al., 2007). Ils améliorent la structure des sols, augmentent la capacité de rétention en eau et des nutriments dans le sol, stimulent l'activité microbienne et augmentent le rendement (Kowaljow et Mazzarino, 2007). La matière organique contenue dans les engrais organiques rend le sol friable, meuble avec une grande porosité, ce qui permet une bonne infiltration de l'eau. Elle tire et relâche les éléments nutritifs, les rendant ainsi accessibles à la plante. La matière organique nourrit et abrite un grand nombre d'organismes utiles du sol, tels que les vers de terre et les microorganismes, qui œuvrent continuellement à l'amélioration de la fertilité et, à la structure du sol (Ouédraogo et al. 2008), d'où

\section{MATERIEL ET METHODES \\ Matériel}

Site expérimental : L'essai a été conduit à la Station d'Expérimentations Agronomiques de Lomé de l'Ecole Supérieure d'Agronomie de l'Université de Lomé, situé sur le cordon littoral à $6^{\circ} 10$ latitude Nord et $1^{\circ} 10$ longitude Est et à 19-60 m au-dessus de la mer. Le terrain de l'essai est assez plat. Les parcelles expérimentales ont été installées sur un sol ferralitique à horizon supérieur sableux de structure grumeleuse dont les horizons de profondeurs sont argileux. Ce sol présente un déficit en matière organique $1,08 \%$, azote $0,05 \%$ et un $\mathrm{pH}$ de 7.73 avec une texture sableuse (Djagoudi, 2005). Ce type de sol ferralitique communément appelé "terres de barre", formé à partir l'importance de l'utilisation des fientes de volailles et du fumier de bovin comme engrais organique. Les engrais organiques augmentent la teneur en matière organique du sol, améliorent la capacité d'échange d'aliments, la conservation croissante de l'eau du sol favorisant des agrégats de sol et protégeant le sol contre l'acidité, l'alcalinité, la salinité, les pesticides et les métaux lourds toxiques (Lorch, 2010). Les exigences de la laitue en sol sont faibles. Les laitues s'adaptent à la plupart des sols pourvu que ceux-ci soient riches en humus et frais. Mais, elles préfèrent les sols légers et fertiles, voire riches en matières organiques et en éléments nutritifs surtout l'azote. La laitue a besoin d'oxygène pour mettre en place ses racines. Ainsi, le sol doit donc être aéré, non tassé et non hydromorphe. II doit aussi disposer d'une réserve utile suffisante. La laitue ne supporte ni l'acidité, ni la salinité du sol ou de l'eau d'irrigation (Chidikofan 2010). Dans la perspective d'améliorer le rendement de la laitue, l'essai s'est penché sur l'efficacité de la combinaison des fertilisants organiques et minéraux (NPK15-15-15 et Urée) sur la culture de la laitue de la variété "Eden» sur les terres de barre du sud Togo. L'objectif principal de l'essai est de développer de nouvelles initiatives de gestion de la fertilité des sols compatibles avec la gestion rationnelle des ressources afin d'évaluer l'efficacité des différentes combinaisons d'engrais sur la productivité de la laitue et d'identifier le meilleur model d'amélioration des conditions de culture de cette plante.

du dépôt continental, couvre une partie des terres arables au Ghana, au Togo, au Bénin et au Nigéria (Kadanga et Sogbédji, 2017). Le site d'expérimentation se situe dans la région maritime où la température moyenne est de $27^{\circ} \mathrm{C}$. La hauteur des précipitations varie entre 800 et $1100 \mathrm{~mm}$ par an. II règne un climat équatorial guinéen dans la zone et il est caractérisé par deux saisons pluvieuses et deux saisons sèches. La grande saison pluvieuse va de mi-mars à mi-juillet $\mathrm{La}$ petite saison pluvieuse s'étend de septembre à minovembre. La grande saison sèche va de mi-novembre à mi-mars et la petite saison sèche, de mi-juillet à fin août. Les données pluviométriques de la zone d'essai sont enregistrées sur la figure 1 


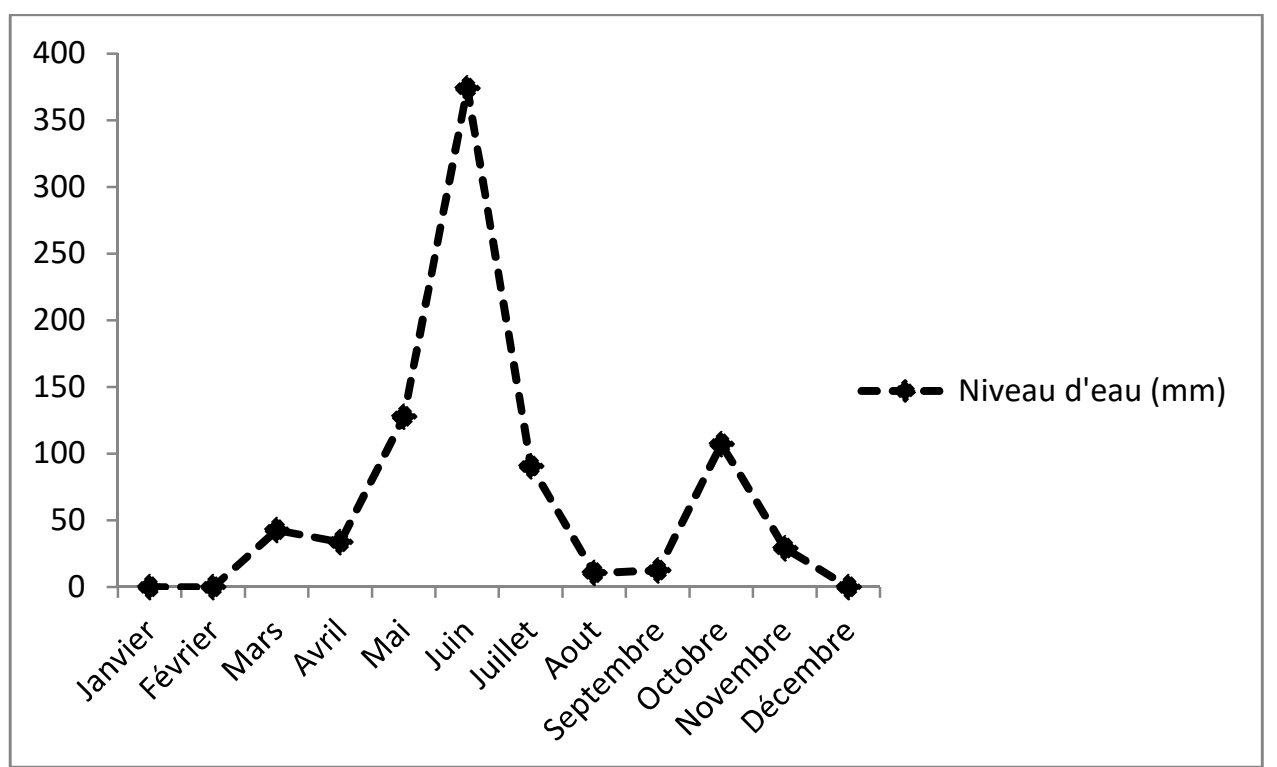

Figure 1 : Courbe des données pluviométriques de la zone d'essai, 2017

Matériel végétal: Le matériel végétal utilisé est constitué des semences de laitue de la variété Eden. Les graines sont de très petites tailles allongées en forme de riz. Elles font 3 à 4 semaines en pépinière et 4 à 6 semaines sur planches. C'est une variété à pomme assez ronde, à feuilles larges, craquantes, ondulés et nervures parallèles. Elle est la plus produite par les jardiniers (Koudigué, 2018).

Fertilisants: Le fumier de bovins et de fiente de volaille utilisé est collecté dans la section Elevage de la Station d'Expérimentations Agronomiques de Lomé de l'Université de Lomé. Les engrais minéraux utilisés sont le NPK 15-15-15 et l'Urée. La fertilisation dans le cadre de l'essai a consisté à apporter 16 tonnes/ha des fumiers des ruminants et des fientes de volailles bien décomposés et de $150 \mathrm{Kg} \mathrm{ha}^{-1}$ de NPK en fumure de fond ainsi que $50 \mathrm{Kg}$.ha-1 ${ }^{-1}$ 'urée en fumure d'appoint.

\section{Méthodes}

Dispositif expérimental : Le dispositif adopté est celui en blocs complètement aléatoires de quatre traitements avec trois répétitions. Le schéma cultural utilisé est $0,35 \mathrm{~m} \times 0,35 \mathrm{~m}$ à raison d'un plant par poquet sous les traitements suivants :

T0 : témoin absolu, sans apport de fertilisants

T1 : apport de 16 t.ha-1 $^{-1}$ de fumier de bovin $+150 \mathrm{Kg}$.ha1 de NPK + $50 \mathrm{Kg}$ ha $^{-1} \mathrm{~d}^{\prime}$ Urée

T2 : apport de 16 tha $^{-1}$ de fientes de volailles +150 Kg.ha-1 de NPK $+50 \mathrm{Kg}^{-h^{-1} \mathrm{~d}^{-1} \text { Urée }}$

T3 : apport de 16 t.ha $^{-1}$ d'un mélange équitable de fumier de bovin et de fientes de volailles (soit $50 \%$ de fumier de bovin et $50 \%$ de fiente de volaille) +150 Kg.ha-1 de NPK + 50 Kg.ha-1 d'Urée.

Les opérations culturales : Le terrain de la culture a été réalisé un mois avant le repiquage suivi d'un labour profond de $15 \mathrm{~cm}$ et du nivelage de façon à respecter les dimensions qui sont de $3,00 \mathrm{~m}$ sur $1,22 \mathrm{~m} ; 0,3 \mathrm{~m}$ entre deux planches et $0,6 \mathrm{~m}$ entre deux blocs. Le semis a été réalisé à faible profondeur. La pépinière a duré 21 jours. Le repiquage en plein champ a été réalisé dans un après-midi sur des planches de 30 plants bien arrosées. Les plants vigoureux, atteints le stade de 3 à 4 feuilles, ont été repiqués. A la fin du repiquage, les jeunes plants ont été convenablement arrosés. L'épandage des engrais organiques a été fait juste après la confection des planches suivant le dispositif expérimental. Le NPK15-15-15 à la dose de

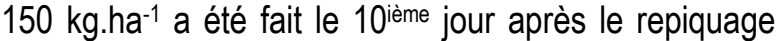
puis le 15 ième jour, l'urée a été épandue à la dose de $50 \mathrm{~kg} / \mathrm{ha}$. L'arrosage qui s'est fait 3 fois par jour après le repiquage et 2 fois par jour après la reprise des plants. Le binage suivi du sarclage des allées ont été effectués 3 fois par mois du repiquage des plants jusqu'à la maturité ce qui a permis de lutter efficacement contre les adventices et ameublir le sol pour une bonne aération et infiltration d'eau. La récolte est faite le 55 ième jour après le semis. Les pesées sont réalisées sur le site sur un échantillon de 5 plants pris de façon aléatoire. Le principe de récolte a consisté à arracher les plants de laitue après arrosage des planches, de rincer le système racinaire afin de prendre les données. Les paramètres de croissance et de développement 
végétatifs étudiés ont concerné le diamètre du couvert végétal des plants, la hauteur des plants (du niveau du sol à la cime de la plante), le nombre de feuilles vertes et ainsi que la détermination de leur masse par plant. Le rendement a été calculé en additionnant les poids des pieds récoltés au niveau de chaque parcelle durant toute la période de la récolte par la formule suivante: $\mathbf{R d t}=(\mathbf{P} \times \mathbf{1 0 . 0 0 0}) / \mathrm{S}$. Rdt : rendement en plant de laitue $\left(\right.$ t.ha $\left.^{-1}\right), \mathbf{P}$ : masse moyenne des plants récoltés

\section{RÉSULTATS}

Effet des fertilisants sur le diamètre moyen du couvert végétal : Le diamètre moyen et la longueur de la tige de la laitue ont été mesurés. Ces observations sont effectuées sur 5 tiges de laitue sélectionnées d'une façon aléatoire. La circonférence de la tige est mesurée au collet puis le diamètre est calculé par la par traitement (t) et $\mathbf{S}$ : surface nutritionnelle d'un plant $\left(m^{2}\right)$

Traitement des données statistiques: Les données obtenues sont saisies avec le tableur Excel et analysées à l'aide du logiciel SPSS (Statistical Package for Social Science) version 21.0 pour une analyse de la variance (ANOVA). Le test de DUNCAN au seuil de 5\% est utilisé pour discriminer les moyennes des différents traitements.

formule : $\mathbf{d}=$ circonférence $I \boldsymbol{\pi}$, où $\pi=3,14$. Par contre la mesure de la longueur est prise du collet à l'extrémité de la tige. La masse de la laitue est déterminée à partir d'une balance électronique de portée $4 \mathrm{~kg}$.

Tableau 1 : Valeurs moyennes du diamètre du couvert végétal des plants par traitement

\begin{tabular}{ll}
\hline Traitements & Diamètre moyen du couvert végétal des plants, $(\mathbf{c m})$ \\
\hline T0 & $32,53 \pm 1,19 \mathrm{a}$ \\
T1 & $38,33 \pm 0,64 \mathrm{~b}$ \\
T2 & $42,80 \pm 0,91 \mathrm{c}$ \\
T3 & $38,80 \pm 0,98 \mathrm{~b}$ \\
F & 19,55 \\
P & 0,001 \\
\hline
\end{tabular}

Le diamètre moyen du couvert végétal, selon les traitements, va de $32,53 \mathrm{~cm}$ à $42,80 \mathrm{~cm}$ (Tableau 1). L'analyse de la variance au seuil de $5 \%$ a montré une différence significative entre le diamètre moyen du couvert végétal des différents traitements. Le test de Duncan réalisé au seuil de $5 \%(P<0.05)$ a permis de regrouper les traitements en trois classes distinctes $(a$; $b$ et $c)$. Selon cette discrimination les plants du témoin T0 présentent un diamètre plus petit, soit une moyenne de $32,53 \mathrm{~cm}$. Les plants du traitement T2 présentent un meilleur diamètre moyen de $42,80 \mathrm{~cm}$ qui dépassent celui des plants du traitement T0 de $31,57 \%$ suivi des diamètres des plants des traitements $\mathrm{T} 3$ et $\mathrm{T} 1$ statistiquement identiques et dépassant respectivement de $19,27 \%$ et $17,83 \%$ celui des plants du traitement TO.
Effets des traitements sur la hauteur des plants : La hauteur moyenne des plants va de $21,13 \mathrm{~cm}$ à 24,60 $\mathrm{cm}$ (Figure 2). L'analyse de la variance au seuil de $5 \%$ $(P<0.05)$ a montré que la différence observée entre les traitements est significative. Le test de Duncan réalisé au seuil de $5 \%$ a permis de regrouper les traitements en deux classes distinctes ( $a$ et $b$ ). Selon cette discrimination le témoin TO a la plus faible hauteur, soit une moyenne de $21,13 \mathrm{~cm}$. Les traitements T1, T2 et T3 présentent des croissances en hauteurs statistiquement identiques, mais arithmétiquement T2 présente une hauteur légèrement plus élevée $(24,60 \mathrm{~cm})$ que celle des autres traitements et surtout plus que celle du témoin T0 avec un gain moyen par rapport au témoin de $16,42 \%$. 


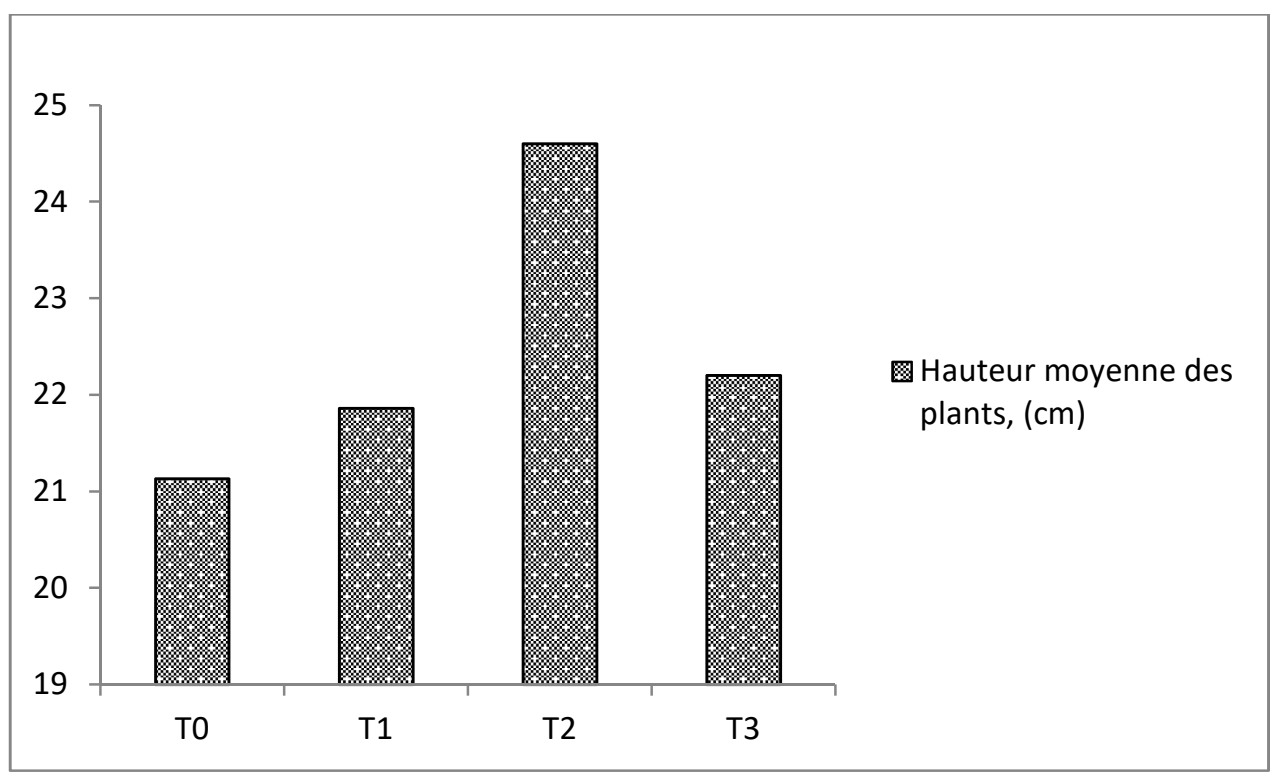

Figure 2 : hauteur moyenne des plants d'ananas par traitement, $(\mathrm{cm})$

Effets des traitements sur le nombre moyen de feuilles par plant

Tableau 2 : Nombre moyens de feuilles par plant par traitement

\begin{tabular}{ll}
\hline Traitements & Nombre moyen de feuilles par plant \\
\hline T0 & $22,00 \pm 1,38 \mathrm{a}$ \\
T1 & $25,53 \pm 0,55 \mathrm{~b}$ \\
T2 & $29,26 \pm 0,92 \mathrm{~b}$ \\
T3 & $26,60 \pm 1,54 \mathrm{~b}$ \\
F & 6,58 \\
P & 0,001 \\
\hline
\end{tabular}

Le nombre moyen de feuilles par plant varie de 22,00 (pour T0) à 29,26 (pour T2) (Tableau 2). L'analyse de la variance au seuil de $5 \%(P<0.05)$ a montré que la différence observée entre les traitements est significative. Le test de Duncan réalisé au seuil de $5 \%$ a permis de regrouper les traitements en deux classes distinctes $(a$ et $b)$ : Selon cette discrimination le témoin TO occupe le plus faible nombre de feuilles, soit une moyenne de 22,00 feuilles par plant. Les traitements
$\mathrm{T} 1(\mathrm{~b}), \mathrm{T} 2(\mathrm{~b})$ et $\mathrm{T} 3(\mathrm{~b})$ présentent des nombres de feuilles par plant statistiquement identiques, mais arithmétiquement T2 présente un nombre moyen de feuilles légèrement élevé $(29,26)$ plus que celui des autres traitements et surtout plus que celui du témoin T0 avec un gain moyen par rapport au témoin de 33\%. Effets des traitements sur le rendement des plants de laitue: Les résultats issus de l'évaluation du rendement de la laitue sont consignés sur la figure 3 . 


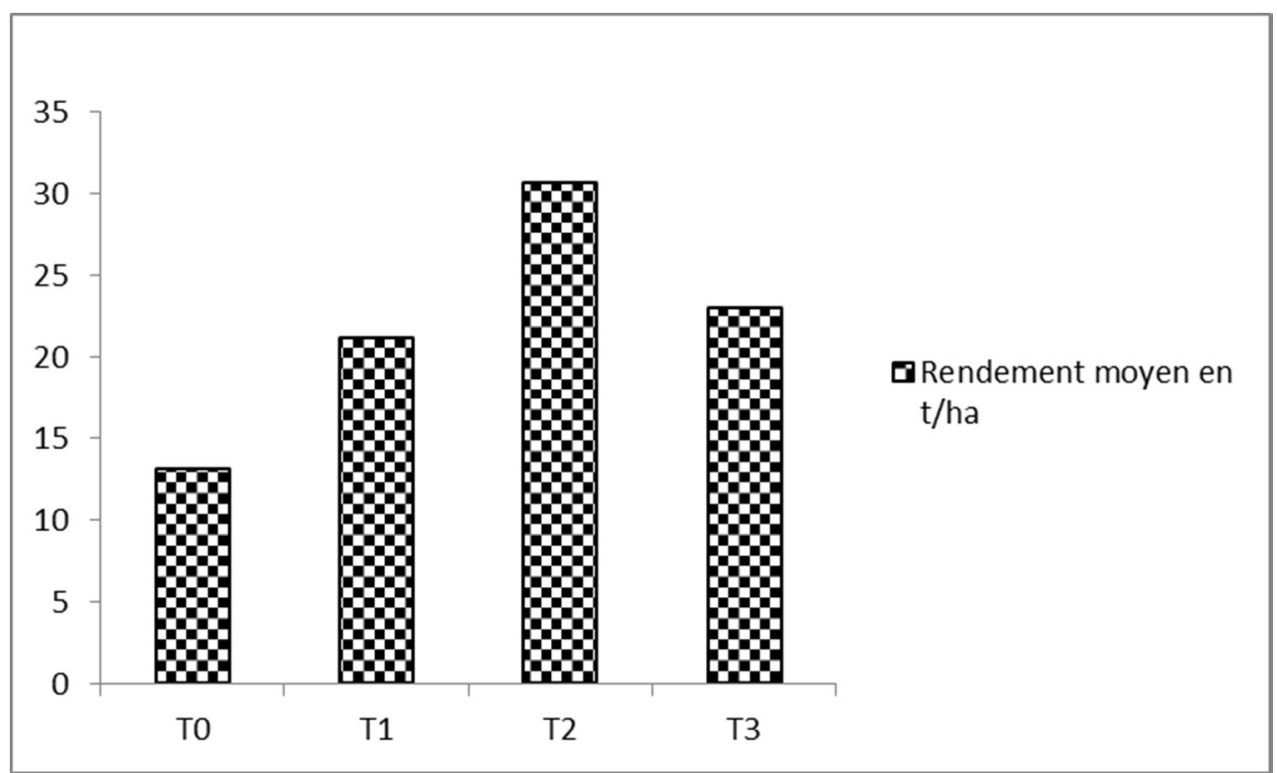

Figure 3 : rendement moyen des plants de laitue par traitement, (t ha-1)

Le rendement de la laitue varie entre 13,08 t.ha $^{-1}$ et 30,60 t.ha $^{-1}$ (Figure 3). L'analyse de la variance au seuil de $5 \%$ a montré que la différence observée entre les traitements est significative. Le test de Duncan réalisé au seuil de $5 \%$ a permis de regrouper les traitements en trois classes distinctes $(a ; b$ et $c)$. Selon cette discrimination le témoin TO(a) occupe la plus basse

\section{DISCUSSION}

Effet des traitements sur le diamètre du couvert végétal : Le diamètre du couvert végétal des plants de laitue sur les parcelles ayant reçu des fertilisants ont dépassé ceux de la parcelle témoin (T0). Ceci montre la pauvreté en éléments nutritifs de la parcelle témoin. D'autre part les fertilisants utilisés sous les autres traitements seraient de bonne qualité et auraient influencé positivement le diamètre du couvert végétal des plants de la laitue. Le $\mathrm{pH}$ de la parcelle expérimentale est de 5,2-6,8 et le rapport $\mathrm{CN}^{-1}$ est de 7,1 (Tossah, 2000). Selon Ondo en 2011 et Ognalaga et al. en 2015, dans leurs travaux d'investigation respectivement sur la vulnérabilité des sols maraîchers du Gabon région de Libreville, cas de l'acidification et la mobilité des éléments métalliques et l'effet des écumes à cannes à sucre, de la poudre et du compost à base de Chromolaena odorata (L.) sur la croissance de l'oseille de Guinée, ont conclu qu'un pH voisin de la neutralité constitue un atout pour une meilleure absorption racinaire des éléments nutritifs. Ce que confirme également la thèse de Baize en 2000 selon laquelle, lorsque le rapport $\mathrm{CN}^{-1}$ est inférieur à 25 , la classe avec un rendement de 13,08t/ha. Les plants du traitement T2(c) présentent le meilleur rendement $\left(30,61\right.$ t.ha $\left.^{-1}\right)$ avec $134 \%$ de gain par rapport au témoin suivi des traitements $\mathrm{T} 3(\mathrm{~b})$ et $\mathrm{T} 1(\mathrm{~b})$ qui sont statistiquement identiques dépassant le témoin respectivement de $75,68 \%$ et de $62,16 \%$.

libération d'ammonium et de nitrates l'emporte sur la réorganisation; et l'alimentation azotée des plantes devient possible à partir des réserves azotées du sol. Cette observation également corrobore celle d'Amadji et al. (2009). L'analyse statistique des données montre que le meilleur diamètre du couvert végétal est enregistré sur les plants du traitement T2. Ce qui a permis aux plants de capter plus de lumière et d'avoir une croissance plus rapide que ceux des autres traitements. La richesse du traitement T2 en azote induit par des fientes de volailles (15 kg/t de N, Jacques et Pierre 2005) confirme ce résultat. Ceci corrobore avec les observations faites par Saïdou et al (2012) après utilisation du compost enrichi avec la fiente de volaille pour la productivité de la laitue. En effet l'azote étant un élément constitutif de la chlorophylle, il est un facteur déterminant dans la croissance et la détermination du rendement des plantes (Magnan, 2006).

Effets des traitements sur le nombre moyen de feuilles et la hauteur moyenne des plants: Le nombre moyen de feuilles et celui de la hauteur des 
plants des parcelles ayant reçu les fertilisants ont dépassé ceux de la parcelle témoin (T0). L'analyse statistique montre que la hauteur moyenne des plants sous l'effet des différents fertilisants est statistiquement identique entre les traitements T1, T2 et T3. Celle du nombre moyen des feuilles est aussi statistiquement identiques entre les traitements T1, T2 et T3. Ceci suggère que les traitements ont eu presque les mêmes effets positifs sur ces deux paramètres. En effet, tous les traitements ont mis à la disposition des plants, au moment opportun les différents éléments nutritifs nécessaires pour la croissance en hauteur et des feuilles des plants. Ces résultats sont conformes à ceux de William en 2003 qui révèlent que l'azote stimule une croissance de la partie aérienne favorisant aussi l'augmentation de rapport tiges feuillées, racines et hauteurs des plants. Stevenson en 1986 a abordé dans le même sens à travers ses travaux qui ont prouvé que l'azote favorise l'utilisation des hydrates de carbones qui stimulent le développement et l'activité racinaire favorisant ainsi l'exportation des autres éléments minéraux et la croissance des plantes. Les résultats obtenus pourraient s'expliquer par la forte teneur de la fiente de volaille en azote $(15 \mathrm{~kg} / \mathrm{t} \mathrm{de} \mathrm{N}, 12 \mathrm{~kg} / \mathrm{t}$ de $P$, et $9 \mathrm{~kg} / \mathrm{t}$ de K) selon Jacques et Pierre en 2005, principal facteur de croissance des végétaux verts. La remarque a été également faite dans le traitement avec la bouse de vache plus fiente de volaille par une augmentation de la taille des plants. Ces résultats corroborent ceux de Tchabi et al, en 2012 qui précisent que la bouse de vache est un engrais de qualité notamment par sa forte teneur en azote. En effet, l'azote est un facteur essentiel de croissance des plants, surtout au niveau des feuilles et tiges. Toute fois la teneur en azote de déjection de volaille $(15 \mathrm{~kg} / \mathrm{t}$ de $\mathrm{N}, 12 \mathrm{~kg} / \mathrm{t}$ de $\mathrm{P}$, et $9 \mathrm{~kg} / \mathrm{t}$ de $\mathrm{K})$ est supérieure à celle de la vache $(6 \mathrm{~kg} / \mathrm{t}$ de $\mathrm{N}, 3$ $\mathrm{kg} / \mathrm{t}$ de $P$, et $6 \mathrm{~kg} / \mathrm{t}$ de $\mathrm{K}$ ) selon Jacques et Pierre en

\section{CONCLUSION}

L'étude a consisté à évaluer l'impact des différentes combinaisons d'engrais organiques et minéraux sur la productivité de la laitue. Les résultats obtenus de l'expérimentation ont monté que le traitement T2 constitué de fumier de fientes de volaille a augmenté le rendement de la laitue suivi du traitement T3 (fumier de mélange d'excréments de bovins et de fientes de

\section{REFERENCES BIBLIOGRAPHIQUES}

Amadji GL., Saîdou A., Chitou L. 2009. Recycling of residues in compost to improve coastal sandy
2005, d'où une croissance en taille remarquable au niveau des plants du traitement $\mathrm{T} 2(24,60 \mathrm{~cm} \pm 0,68)$. Effets des traitements sur le rendement des plants de laitue : Les rendements des parcelles ayant reçus des fertilisants ont dépassé ceux de la parcelle témoin (T0). Ceci suggère que la combinaison de fertilisants apportés sous la culture a eu des effets positifs sur les rendements. Dans leurs travaux de recherches, Dakouo et al. en 1995 ; Bado en 2002 et Mills et al. en 2003 ont, chacun, montré que l'association fumier et engrais minéraux donne les meilleurs rendements. La comparaison des rendements obtenus des traitements T1 $(21,21 \pm 15,08$ (b) t.ha-1), T $2(30,61 \pm 24,38$ (c) t.ha-1) et T3 $\left(22,98 \pm 24,38\right.$ (b) t.ha $\left.{ }^{-1}\right)$ montre une différence significative entre ces résultats qui pourrait s'expliquer par une différence entre les bilans en éléments nutritifs apportés par chaque traitement. Or les trois traitements ont reçu la même quantité de NPK et d'Urée. Alors la différence significative ne peut être due qu'aux différents fertilisants organiques apportés et à leurs caractéristiques biologique et physico-chimique. Le rendement le plus élevé obtenu en T2 $(30,61 \pm 24,38$ t.ha- $^{-1}$ ) pourrait s'expliquer par la dégradation rapide et la richesse des fientes de volaille en éléments minéraux et surtout en azote que le fumier de bovins (Christophe, 2004). La laitue étant une plante à cycle court, elle profite des nutriments de cette dégradation rapide des fientes. Ceci corrobore les observations faites par Amadji et al. en 2009 après utilisation du compost enrichi avec la fiente de volaille pour la production de choux sur sol sableux. Les mêmes résultats ont été obtenus par Saïdou et al. en 2012 après utilisation du compost enrichi avec la fiente de volaille pour la production de laitue sur un sol ferralitique. L'azote étant un élément constitutif de la chlorophylle, il est un facteur déterminant dans la croissance et la détermination du rendement des plantes (Magnan, 2006).

volailles) puis du traitement T1 (fumier d'excréments de bovin) qui ont donné respectivement un surplus de produit de $134,00 \%, 75,69 \%$ et de $62,16 \%$ par rapport au traitement témoin (T0). La laitue étant une plante à cycle court, l'utilisation du fumier à base de fientes de volailles, riche en azote dont la libération est rapide, serait mieux indiquée à cette culture.

soil properties and cabbage shoot yield in Bénin. Int J Biol chem sci, 3(2) : 192-202. 
Amouzouvi K. A. A. Adjétey-Bahum F. A., Dansou T. A. 2005. Effet des émondes de quelques espèces de légumineuses arborescentes sur la fertilité du soil et le rendement des cultures vivrières dans un système agroforestier : cas du soja. Ecole Supérieure d'Agronomie de I'Université de Lomé. J. Rech. Sci. Univ. Lomé (Lomé (Togo), 2005, série A, 7(1), 39-45

Ayebou G. (2003). Effet du mulch de quelques arbres agroforestiers sur le rendement en grains de maïs au sud du Togo. Mémoire d'Ingénieur Agronome ESA- UL, 60p.

Bado B.V. 2002. Rôle des légumineuses sur la fertilité des sols ferrugineux tropicaux des zones guinéenne et soudanienne du Burkina Faso. Thèse de doctorat : Université de Laval (Québec) ,197p.

Baize D. 2000. Guide des Analyses en Pédologie (2nd edn). INRA : Paris Magnan J. 2006. Epandage post récolte des engrais organiques et risques environnementaux reliés aux pertes d'azote. Ordre des Agronomes du Québec., p.75.

Biaou O. D. B., Saïdou A. Bachabi F-X Padonou, G. E. et Balogoun 2017. Effet de l'apport de différents types d'engrais organiques sur la fertilité du sol et la production de la carotte (Daucus carota L.) sur sol ferralitique au sud Bénin. International Journal of Biological and Chemical Sciences, 11(5), p. 2316

Chidikofan Grâce D. M. F. 2010. Contribution à l'amélioration de la qualité des cultures maraîchères du site de Houéyiho à Cotonou au Bénin : Cas de la laitue (Lactuca sativa L.). Mémoire pour l'obtention du Master en Ingénierie de l'eau et de l'Environnement. Option : Environnement, P. 7.

Christophe J. 2004. Bouse: historique, importance et écosystème. Thèse de doctorat vétérinaire, université de Toulouse, $82 p$.

Dakouo D. ; Koulibaly R ; Hien V., 1995. Agronomie et techniques culturales. Rapport de la Campagne, 1993-1994. 62 p.

Dansou A. 2003. Influence des émondes de quelques arbres agroforestiers sur la fertilité des soils et le rendement en graines du soja. Mémoire d'Ingénieur Agronome de l'Ecole Supérieure d'Agronomie de l'Université de Lomé. Option Phytotechnie, p. 26-28

Djagoudi M. 2005. Effets de l'utilisation de l'urine humaine hygiénisée sur les cultures maraîchères et les propriétés du sol : cas de la culture de laitue, de chou pomme et de tomate. (Mémoire d'Ingénieur Agronome de l'Ecole Supérieure d'Agronomie de l'Université de Lomé. Option Phytotechnie. P. 15.

IFDC. 2013. Main streaming pro-poor fertilizer access and innovative practices in West Africa. IFAD Technical Assistance Grant No. 1174 reports. Muscle Shoals, Alabama, U.S.A.

Jacques Petit et Pierre Jobin, 2005.La fertilisation organique des cultures. Les bases. La Fédération d'Agriculture Biologique du Québec (FABQ). P. 26

Kadanga P. et Sogbédji, 2017. Journal de Recherche Scientifique de l'Université de Lomé No 2 Spécial P. 23.

Koné B, Diatta S, Saïdou A, Akintayo I, Cissé B. 2009. Réponses des variétés interspécifiques du riz de plateau aux applications de phosphate en zone de forêt au Nigeria. Canadian Journal of Soil Science, 89: 555-565. DOI: https://doi.org/10.4141/CJSS08086.

Koudigué R. C. Y., 2018. Effets combinés des fertilisants organiques, du NPK 15-15-15 et de l'Urée sur la productivité de la laitue (Lactuca sativa L.) : cas des sols ferralitiques du sud Togo. Mémoire de Licence en Sciences Agronomiques. Mention Phytotechnie. $54 \mathrm{P}$.

Kowaljow E, Mazzarino MJ. 2007. Soil restoration in semarid Patagonia: chemical and biological response to different compost quality. Soil Biological. Biochemistry, 39: 1580-1588.

Lorch A. 2010. Les Micro-organismes efficaces au quotidien : Au service de la terre, des animaux et des hommes, Le Souffle D'OR. ed. 382 p.

Macauley $H$. et Ramadjita T., 2015. Les cultures céréalières rir, mais millet sorgho et blé. Document de référence $21-23$ octobre : $38 p$.

Magnan J. 2006. Epandage post récolte des engrais organiques et risques environnementaux reliés aux pertes d'azote. Ordre des Agronomes du Québec., p.75.

Mills A.J. \& Fey M.V., 2003. Declining soil quality in South Africa : effects of land use on soil organic matter and surface crusting. South Afr. 1. ScL, 99, 429-436.

Ognalaga M, Odjogui PIO, Lekambou JM, Poligui RN. 2015, Effet des écumes à cannes à sucre, de la poudre et du compost de à base de Chromolaena odorata (L.) King R.M. \& H.E. Rob sur la croissance de l'oseille de Guinée 
(Hibiscus sabdariffa L.). Int. J. Biol. Chem. Sci., 9(5) : 2507-2519.

Ondo JA, 2011. Vulnérabilité des sols maraîchers du Gabon région de Libreville: acidification et mobilité des éléments métalliques. Thèse Université de Provence, France, 113-128.

Ouédraogo, A., Yombi, L., Doumbia, S., Eyhorn, F., DISCHL, R. 2008. Guide de production du coton biologique et équitable (manuel de référence pour l'Afrique de l'Ouest). Helvetas. $49 \mathrm{p}$.

Saïdou A, Bachabi SFX, Padonou GE, Biaou ODB, Balogoun I, Kossou D. 2012. Effet de l'apport d'engrais organiques sur les propriétés chimiques d'un sol ferralitique et la production de laitue au Sud Bénin. Rev. CAMES-Série A., 13(2) : 281-285.

Stevenson, J.F. 1986. Cycles of soil : carbon, nitrogen, phosphorus, sulfur, micronutrients. John Wiley \& Sons, New York

Tchabi VI, Azocli D, Biaou GD. 2012. Effet de différentes doses de vache sur le rendement de la laitue (Lactuca sativa L.). Int. J. Biol. Chem. Sci., 6(6): 50785084.

Tossah B. K. 2000. Influence of soil properties and organic inputs on phosphorus cycling in herbaceous legume-based cropping systems in the West African derived savanna Ph.D. Thesis No. 428 K.U. Leuven. Belgium.

Weber J, Karczewska A, Drozd J, Lieznar M, Lieznar S, Jamroz E, Kocowiez A. 2007. Agricultural and ecological aspects of sandy soil as affected by the application of municipal solid waste composts. Soil Biology and Biochemistry, 39: 1294-1302

William G., 2003 : Physiologie végétale, Editions De Boeck Université, rue des Minimes 39, B-1000 Bruxelles, 110-115p. 